OPEN ACCESS

Edited by:

José Manuel Pérez-Pérez,

Miguel Hernández University of Elche,

Spain

Reviewed by:

Juan Xu,

Zhejiang University, China

Tong Zhang,

Pacific Northwest National Laboratory

(DOE), United States

Hoo Sun Chung,

Ghent University Global Campus,

South Korea

${ }^{*}$ Correspondence:

Ive De Smet

ive.desmet@psb.vib-ugent.be

Specialty section:

This article was submitted to Plant Development and EvoDevo,

a section of the journal

Frontiers in Plant Science

Received: 18 June 2020

Accepted: 27 July 2020

Published: 07 August 2020

Citation:

Pan L and De Smet I (2020) Expanding the Mitogen-Activated Protein Kinase (MAPK) Universe:

An Update on MAP4Ks.

Front. Plant Sci. 11:1220.

doi: 10.3389/fp/s.2020.01220

\section{Expanding the Mitogen-Activated Protein Kinase (MAPK) Universe: An Update on MAP4Ks}

\author{
Lixia Pan ${ }^{1,2}$ and Ive De Smet ${ }^{1,2 *}$ \\ 1 Department of Plant Biotechnology and Bioinformatics, Ghent University, Ghent, Belgium, 2 VIB Center for Plant Systems \\ Biology, Ghent, Belgium
}

Phosphorylation-mediated signaling cascades control plant growth and development or the response to stress conditions. One of the best studied signaling cascades is the one regulated by MITOGEN-ACTIVATED PROTEIN KINASEs (MAPKS). However, MITOGENACTIVATED PROTEIN KINASE KINASE KINASE KINASEs (MAP4KS) are hardly explored. Here, we will give a comprehensive overview of what is known about plant MAP4Ks and highlight some outstanding questions associated with this largely uncharacterized class of kinases in plants.

Keywords: signaling, kinases, MAP4Ks, phosphorylation, development, stress

\section{INTRODUCTION}

To regulate their growth and development or respond to stress conditions, plants use-like many other organisms-phosphorylation-mediated signaling cascades. The central enzymes in these cascades are kinases, which-via reversible phosphorylation-mediate protein folding (conformation), protein function and the regulation of enzymatic activities, define substrate specificity, and influence protein localization, complex formation, and degradation (Stone and Walker, 1995). The Arabidopsis genome contains more than 1,000 kinases (Wang et al., 2003; Wang et al., 2007; Dissmeyer and Schnittger, 2011), including a largely uncharacterized class of mitogenactivated protein kinase kinase kinase kinases (MAP4Ks) (Champion et al., 2004). Here, we will give a comprehensive overview of what is known about plant MAP4Ks and highlight some outstanding questions.

\section{THE CANONICAL MAPK SIGNALING MODULE}

One of the best studied signaling cascades is the one regulated by MITOGEN-ACTIVATED PROTEIN KINASEs (MAPKs). The MAPK cascade is conserved in yeast, insects, nematodes, plants, and mammals, and its main role is to modulate protein function through linear sequential serine/threonine and/or tyrosine phosphorylation (Leprince et al., 1999; Dan et al., 2001; Champion et al., 2004; Cakir and Kilickaya, 2015). The canonical MAPK signaling module is composed of a MAPK KINASE KINASE (MAP3K), a MAPK KINASE (MAP2K), and a MAPK (Dan et al., 2001; Colcombet and Hirt, 2008) (Figure 1A). In a typical MAPK cascade, a MAP3K specifically activates a dual specific MAP2K by phosphorylation, which in turn activates a MAPK by phosphorylation of 
threonine and tyrosine residues (Xu and Zhang, 2015; Krysan and Colcombet, 2018). Given the number of MAP3Ks, MAP2Ks, and MAPKs in Arabidopsis thaliana (Jonak, 2002; Champion et al., 2004; Colcombet and Hirt, 2008; Krysan and Colcombet, 2018), the theoretical MAPK network contains an enormous number of possible combinations.

Plant MAPK modules play important roles in regulating growth and development as well as responses to biotic and abiotic stresses (Mishra et al., 2006; Xu and Zhang, 2015). The sensors/receptors responsible for the activation of most plant MAPK modules are unknown (Xu and Zhang, 2015; Krysan and Colcombet, 2018). However, plant MAPK cascades are key modules downstream of receptor-like protein kinases (RLKs) or G-proteins (Cho et al., 2008; Cheng et al., 2015; Meng et al., 2015a; Liu and Zhou, 2018; Zhu et al., 2019). Related to developmental processes in Arabidopsis, HAESA (HAE) and HAESA-LIKE 2 (HSL2) function upstream of the MKK4/ MKK5-MPK3/MPK6 module to regulate floral abscission and lateral root emergence (Cho et al., 2008; Zhu et al., 2019) and, for stomata development, ERECTA family members, SOMATIC EMBRYOGENESIS RECEPTOR KINASEs (SERKs) and TOO
MANY MOUTHS (TMM) form a receptor complex that activates-upon ligand perception-the YDA-MKK4/MKK5MPK3/MPK6 module, which leads to phosphorylation of the transcription factor SPEECHLESS (SPCH) (Lau and Bergmann, 2012; Meng et al., 2015b) (Figure 1A). With respect to biotic stress in Arabidopsis, FLAGELLIN-SENSITIVE 2 (FLS2) in a complex with BRASSINOSTEROID INSENSITIVE 1ASSOCIATED KINASE 1 (BAK1) promotes the MAPKKK8MKK1/2-MPK4 cascade and the receptor-like cytoplasmic kinase BRASSINOSTEROID-SIGNALING KINASE 1 (BSK1) directly associating with the FLS2-BAK1 complex, and the MAPKKK5-MKK4/MKK5-MPK3/MPK6 cascade controls the response to flg22 (Chinchilla et al., 2007; Sopena-Torres et al., 2018; Yan et al., 2018; Yu et al., 2018). In the context of abiotic stress in Arabidopsis, CALCIUM/CALMODULINREGULATED RECEPTOR-LIKE KINASE 1 (CRLK1) and CRLK2 are activated by both chilling and freezing temperatures, which leads to rapid MAPKKK8 activation and sequential activation of MKK1/2 and MPK4. The CRLK1/2MAPKKK8-MKK1/2-MPK4 pathway positively regulates cold stress responses by inhibiting the activity of MPK3 and MPK6,

A

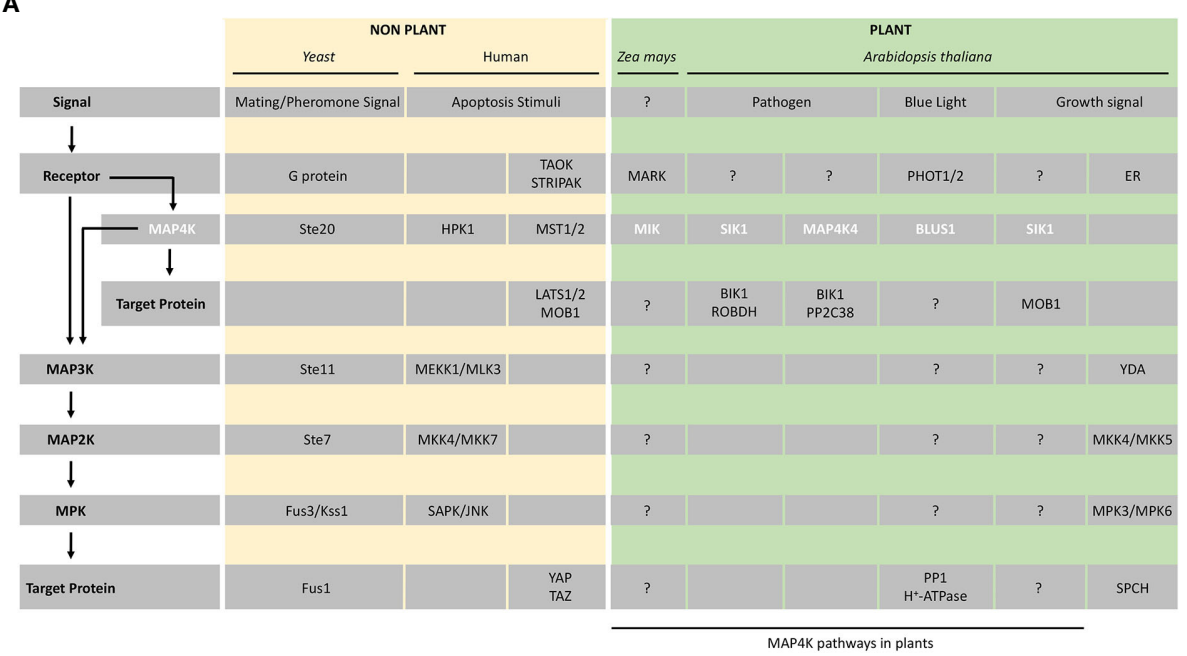

B

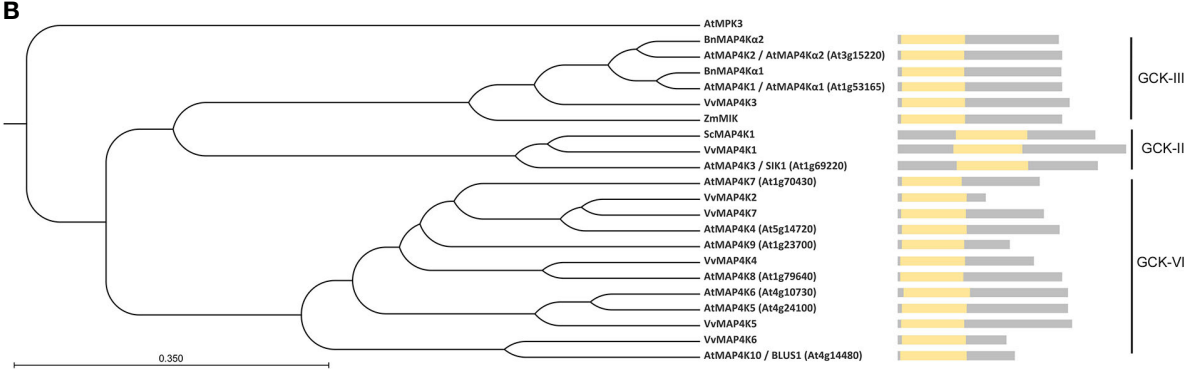

FIGURE 1 | MAP4Ks in plants. (A) Representative examples of MAP4Ks in non-plants (pale gold) and plant (green) examples discussed in the text and their targets and signaling pathways. General protein classes, upstream signal, and possible regulatory pathways (arrows) are indicated on the left and relate to the specific protein names in the gray rows. Plant MAP4Ks are indicated in white.?, indicates missing knowledge (upstream regulation and/or downstream targets) in our understanding of indicated MAP4K pathways. (B) Phylogenetic tree of MAP4Ks in Arabidopsis and orthologs in other species discussed in the text. The tree was built using the UPGMA method and the Jukes-Cantor model (bootstrap analysis with 100 replicates) in the CLC Main Workbench 20.03 (CLC Bio-Qiagen, Aarhus, Denmark). A schematic of the protein sequence is shown, including the position of the kinase domain (yellow). The related subclass within the germinal center kinase (GCK) family is indicated. 
which phosphorylate and promote the degradation of the transcription factor Inducer of CBF Expression 1 (ICE1) (Zhao et al., 2017; Liu and Zhou, 2018). Finally, RACK1 proteins, such as RACK1A, RACK1B, and RACK1C, function as MAPK scaffold proteins and link upstream $\mathrm{G}$ proteins to a downstream MAPKKK8-MKK4/MKK5-MPK3/MPK6 module in PrpL/ ArgC protease-triggered immune signaling in Arabidopsis (Cheng et al., 2015; Meng et al., 2015a; Su et al., 2015).

\section{A NEW HOPE: THE MAP4K FAMILY IN PLANTS}

However, in contrast to the activation mechanisms described above, a MAP3K is sometimes phosphorylated by a MAP4K (Dan et al., 2001; Champion et al., 2004) (Figure 1A). For example, yeast Ste20 acts as a MAP4K that directly phosphorylates Ste11, a MAP3K in the mating pathway (Wu et al., 1995), the mammalian Hematopoietic progenitor kinase (HPK1) phosphorylates the MAP3K MEKK1 (Dan et al., 2001; Chuang et al., 2016), and PAK2 activates the MAP3K Raf-1 by direct phosphorylation (Dan et al., 2001; Chuang et al., 2016). The yeast Ste20-related MAP4K family is evolutionarily conserved (Wu et al., 1995; Champion et al., 2004). In non-plant organisms, the MAP4K family is divided in two families according to the location of the kinase domain: (i) p21-activated kinases (PAKs), with a C-terminal kinase domain and an N-terminal GTPase-binding domain, and (ii) germinal center kinase (GCKs), with an N-terminal kinase domain and lacks the GTPase-binding domain (Dan et al., 2001).

However, to complicate matters, some MAP4Ks act as a MAP3K or phosphorylate proteins outside a MAPK module (Figure 1A). For example, in humans, the MAP4Ks Thousandand-one Amino Acid 1 (TAO1) and TAO2 phosphorylate MKK3 and MKK3 or MKK6, respectively, to activate the p38 MAPK pathway (Dan et al., 2001; Chen et al., 2003), and in the mammalian Hippo signaling pathway, the MAP4Ks Mammalian STE20-like 1 (MST1) and MST2 phosphorylate and activate the Large Tumor Suppressor 1 (LATS1)/LATS2-MOB Kinase Activator 1A (MOB1A)/MOB1B complex to regulate cell proliferation, migration, and survival (Plouffe et al., 2016; Bae and Luo, 2018; Chen et al., 2019).

In plants, MAP4Ks were initially identified and described in Brassica napus (Leprince et al., 1999) and later also in Zea mays, Solanum chacoense, and Vitis vinifera (Llompart et al., 2003; Castells et al., 2006; Major et al., 2009; Cakir and Kilickaya, 2015).

In Brassica napus, BnMAP4K $\alpha 1$ and $B n M A P 4 K \alpha 2$ were isolated from a globular/heart stage embryo cDNA library (Leprince et al., 1999). These two putative proteins contain 12 serine/threonine protein kinase catalytic subdomains at the $\mathrm{N}$ terminus, possess long disordered sequences at the C-terminus, and are similar to the GCK subfamily of yeast Ste20-like MAP4Ks (Leprince et al., 1999) (Figure 1B). BnMAP4K $\alpha 1$ and $B n M A P 4 K \alpha 2$ are mainly expressed in flower buds, siliques, different stages of embryogenesis, and roots (Leprince et al., 1999).
MAIZE ATYPICAL RECEPTOR KINASE (MARK)INTERACTING KINASE (MIK), a GCK-III subfamily MAP4K from Zea mays, possesses an N-terminal kinase domain that displays high similarity to the GCK subfamily of yeast Ste20-like MAP4Ks (Llompart et al., 2003) (Figure 1B). Moreover, the kinase domain of MIK contains a VGTPFWMAPEV sequence, which aligns with the signature motif of Ste20-like kinases (Dan et al., 2001; Llompart et al., 2003), and both the N-terminal kinase domain and the C-terminal part share high sequence similarity with AtMAP4K $\alpha 1$, AtMAP4K $\alpha 2$, BnMAP4k $\alpha 1$ and BnMAP4ko2 (Llompart et al., 2003). MIK interacts with MARK, which is an atypical receptor kinase expressed during embryogenesis and in the meristems of adult maize plants, and this interaction increases the activity of MIK (Llompart et al., 2003; Castells et al., 2006) (Figure 1A). Different isoforms of MIK show variable kinase activity and are differentially activated through the interaction with MARK, suggesting that the kinase activity of MIK is also regulated by alternative splicing (Castells et al., 2006).

In Solanum chacoense, ScMAP4K1 is expressed during fertilization and early embryogenesis (Major et al., 2009). Phylogenetic analysis revealed that ScMAP4K1 belongs to the GCK-II subfamily and is the ortholog of Arabidopsis SIK1/ MAP4K3 (Major et al., 2009). However, unlike the GCK-II members in mammals, ScMAPK1 has a central kinase domain (Major et al., 2009). While ScMAP4K1 is strongly expressed in reproductive tissues (such as pollen and pollen tubes) (Wu et al., 1995), full length ScMAP4K1 is not present in both pollen and pollen tubes, suggesting that ScMAP4K1 is also regulated by alternative splicing, similar to MIK in maize (Castells et al., 2006; Major et al., 2009). Interestingly, ScMAP4K1 RNAi lines show altered ovule, seed and fruit development, indicating that ScMAP4K1 plays a vital role in those processes (Major et al., 2009).

\section{THE ARABIDOPSIS MAP4KS AWAKEN}

There are 10 MAP4Ks in Arabidopsis (Champion et al., 2004), but while plant MAP4Ks have been described already at the end of the $20^{\text {th }}$ century, it is only since 2013 that functional characterization in A. thaliana has begun (Takemiya et al., 2013a, Xiong et al., 2016). Most of the Arabidopsis MAP4Ks have an N-terminal catalytic kinase domain, but SERINE/ THREONINE KINASE 1 (SIK1)/MAP4K3 has a more centrally located kinase domain (Xiong et al., 2016; Zhang et al., 2018) (Figure 1B). Phylogenetic analysis based on the MAP4K kinase domain showed that MAP4K $\alpha 1$ and MAP4K $\alpha 2$ are GCK-III subfamily members, that SIK1 belongs to the GCKII subfamily, and that other Arabidopsis MAP4Ks are part of the GCK-VI subfamily (Major et al., 2009) (Figure 1B).

To control stomata opening, phototropins (PHOT1 and PHOT2) activate $\mathrm{H}^{+}$-ATPase, through PROTEIN PHOSPHATASE 1 (PP1) (Kinoshita and Shimazaki, 1999; Kinoshita et al., 2001; Takemiya et al., 2006, Takemiya et al., 2013b). Genetic and biochemical analyses revealed that PHOT1/2 
phosphorylate BLUE LIGHT SIGNALING 1 (BLUS1)/MAP4K10 at the conserved Ser-348 in response to blue light, and this phosphorylation is important for BLUS1 function to regulate blue light-induced stomata opening (Schnabel et al., 2018; Takemiya et al., 2013a) (Figures 1A and 2A). The blus1 mutant stomata do not open in response to blue light but respond to an $\mathrm{H}^{+}$-ATPase activator. Furthermore, blue light-induced phosphorylation of BLUS1 at Ser-348 is absent in the phot1 phot2 double mutant, and a BLUS1 phosphorylation dead (S348A) protein variant cannot complement the blus1 mutant phenotype. Interestingly, an inhibitor of PP1, which mediates the signaling between PHOT1/2 and $\mathrm{H}^{+}$-ATPase, suppresses $\mathrm{H}^{+}$-ATPase phosphorylation but does not affect BLUS1 phosphorylation, suggesting that BLUS1 precedes PP1 in the signaling pathway leading to stomatal opening (Takemiya et al., 2013a; Takemiya and Shimazaki, 2016). However, there is likely also a BLUS1-independent pathway regulating $\mathrm{H}^{+}$-ATPase activity downstream of PHOT1/2 (Kostaki et al., 2020).

Arabidopsis SERINE/THREONINE KINASE 1 (SIK1)/ MAP4K3 encodes a MAP4K kinase that contains a central kinase domain and that is highly conserved in land plants (Figure 1B) (Zhang et al., 2018). SIK1 complements the ste20A yeast mutant phenotypes with respect to bud site selection and mitotic exit (Xiong et al., 2016), confirming that SIK1 is similar to Ste20 and belongs to the MAP4K family (Xiong et al., 2016).
SIK1 is strongly expressed in mature organs and tissues, such as developed vascular tissues, stipules of true leaves, mature trichrome, and guard cells (Xiong et al., 2016). The sik1 mutants are dwarfed and grow slow compared to wild type and display a shorter primary root and shorter root hairs, a reduced rosette leaf area, reduced area and number of the petal cells of fully opened flower, and reduced plant height, smaller siliques, and smaller seeds (Xiong et al., 2016). The abovementioned sik1 mutant phenotypes are caused by a reduced cell number and size, indicating that SIK1 influences growth via regulating cell proliferation and cell expansion (Xiong et al., 2016). In agreement with this, the expression of SIK1 orthologs $B n M A P 4 K \alpha 1$ and $B n M A P 4 K \alpha 2$ is cell cycle-dependent and follows the same expression pattern as a G2-phase marker (Leprince et al., 1999). Thus, resembling the function of Ste20, SIK1 plays a role as a positive regulator of cell cycle exit (Xiong et al., 2016).

Interestingly, the $\mathrm{N}$-terminal part of SIK1 interacts with $\mathrm{MOB}$ KINASE ACTIVATOR 1A (MOB1A) and MOB1B (Xiong et al., 2016). In mammals, Mob1 is involved in cell proliferation and tumor suppression and controls appropriate cell numbers and organ size (Pan, 2010). Also in Arabidopsis, MOB1A regulates organ growth, root tip tissue patterning, cell number and size, and sporophyte and gametophyte development (Galla et al., 2011; Pinosa et al., 2013). SIK1 is localized at the plasma
A

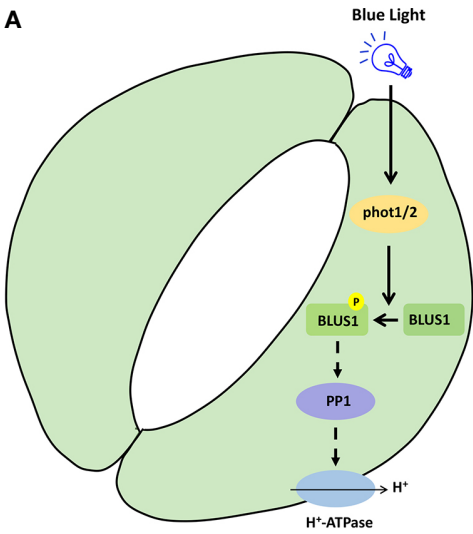

C
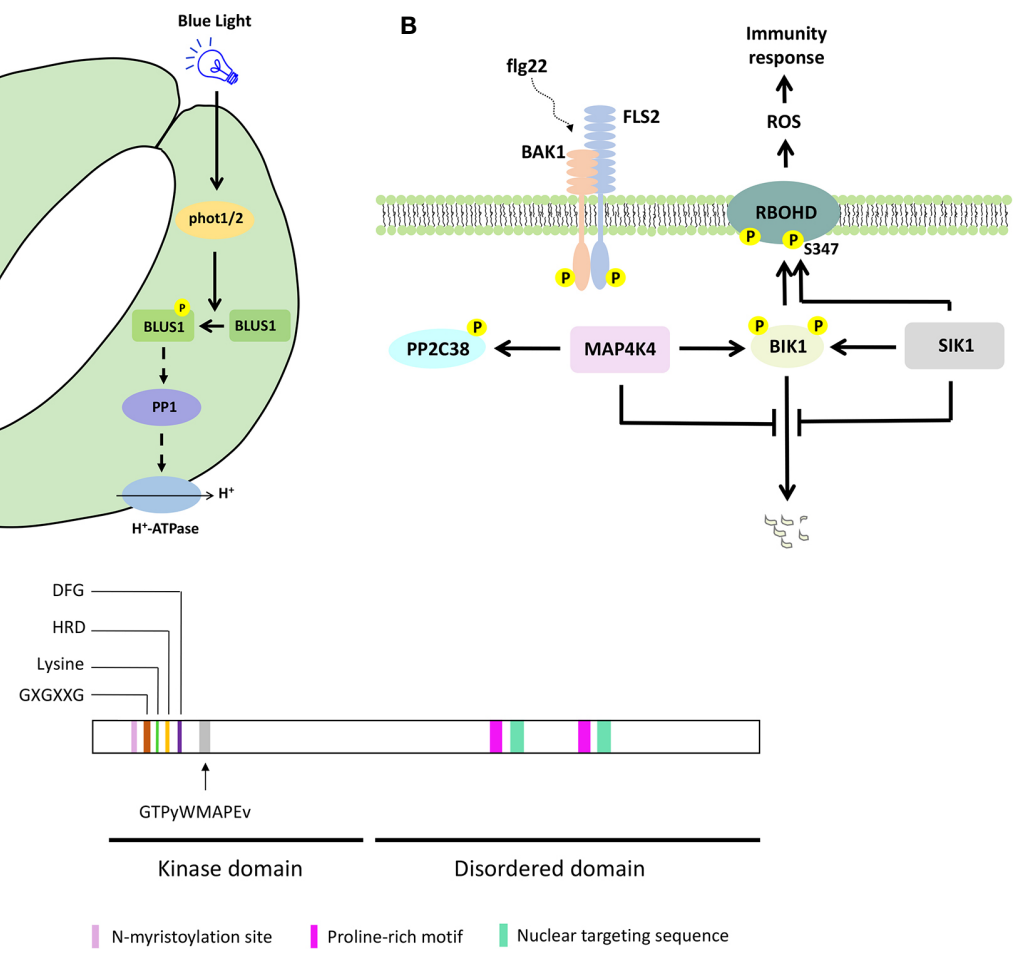

FIGURE 2 | Selected MAP4K signaling pathways and protein features. (A) BLUS1/MAP4K10 signaling to control blue light-induced stomatal opening. (B) Simplified SIK1/MAP4K3 and MAP4K4 signaling to control immunity response. (C) MAP4K protein features on a hypothetical protein sequence. The kinase and disordered domain are indicated, together with the key features in the kinase domain and the consensus sequence associated with a MAP4K. Proline-rich motif, nuclear targeting sequence, and N-myristoylation site were shown for BnMAP4K1 and BnMAP4K2. 
membrane and in the trans-Golgi network/early endosome (TGN/EE), and the interaction of SIK1 and MOBs results in translocation of SIK1 to the nucleus (Xiong et al., 2016).

In humans, MAP4Ks are involved in immunity and activate nuclear factor $\mathrm{kB}$ immune signaling (Chuang et al., 2016). Indeed, also in Arabidopsis, MAP4Ks such as SIK1 and MAP4K4 function in immunity signaling (Zhang et al., 2018; Jiang et al., 2019) (Figures 1A and 2B). Both SIK1 and MAP4K4 directly interact with, phosphorylate, and stabilize the immunity regulator BOTRYTIS-INDUCED KINASE 1 (BIK1) (Zhang et al., 2018; Jiang et al., 2019). Moreover, SIK1 directly interacts with and phosphorylates RBOHD upon flg22 perception (Zhang et al., 2018). In addition, PP2C38, a protein phosphatase, dephosphorylates BIK1 and maintains a minimum BIK1 phosphorylation status in the absence of flg22 (Couto et al., 2016). MAP4K4, which is localized in the cytosol and at the plasma membrane, phosphorylates $\mathrm{PP} 2 \mathrm{C} 38$ in the presence of flg22, and phosphorylated PP2C38 disassociates from BIK1, indicating that MAP4K4 also regulates BIK1 activity through controlling PP2C38 (Jiang et al., 2019).

\section{THE RISE OF MAP4K FEATURES}

Plant MAP4Ks obviously contain several kinase-specific features, such as (i) an invariant lysine residue, an HRD motif, and a DFG motif, which contribute to ATP binding and regulate the catalytic activity of the protein kinase (Carrera et al., 1993; Jiang et al., 2019; Takemiya et al., 2013a; Xiong et al., 2016; Zhang et al., 2018), (ii) a glycine-rich loop with a GXGXXG motif, a structural hallmark of protein kinases (Zhang et al., 2018) (Figure 2C). In addition, the GTPyWMAPEv motif (a small letter indicates less conservation) in subdomain VIII of the kinase domain, termed the Ste20 signature sequence, is the primary reason to classify them as MAP4Ks (Dan et al., 2001; Jonak, 2002; Llompart et al., 2003) (Figure 2C). Furthermore, most plant MAP4Ks possess a long C-terminal region that is largely disordered (Champion et al., 2004; Major et al., 2009) (Figure 2C). Ste20 kinases also often contain proline-rich motifs (Leprince et al., 1999; Chuang et al., 2016; Miller et al., 2019), and these were also described for BnMAP4K1 and BnMAP4K2 (Leprince et al., 1999). While some non-plant MAP4Ks contain a C-terminal citron-homology domain (Chuang et al., 2016), this was not reported for plant MAP4Ks.

BnMAP4K $\alpha 1$ and BnMAP4K $\alpha 2$ also contain an $\mathrm{N}$ myristoylation site (GxxxS/Txxx) between residues 12 and

\section{REFERENCES}

Bae, S. J., and Luo, X. (2018). Activation mechanisms of the Hippo kinase signaling cascade. Biosci. Rep. 38:BSR20171469. doi: 10.1042/BSR20171469

Cakir, B., and Kilickaya, O. (2015). Mitogen-activated protein kinase cascades in vitis vinifera. Front. Plant Sci. 6:556. doi: 10.3389/fpls.2015.00556

Carrera, A. C., Alexandrov, K., and Roberts, T. M. (1993). The conserved lysine of the catalytic domain of protein kinases is actively involved in the phosphotransfer reaction and not required for anchoring ATP. Proc. Natl. Acad. Sci. U. S. A. 90, 442446. doi: 10.1073/pnas.90.2.442
19, which could imply membrane association, and two conserved SH3 binding domains at the C-terminus with proline-rich sequences that can bind SH3-containing adaptor proteins (Leprince et al., 1999) In addition, BnMAP4K $\alpha 1$ possesses two nuclear targeting sequences (PQSRERR and RRGNARERLGNGKVNKR) (Leprince et al., 1999) (Figure 2C).

\section{CONCLUSION AND PERSPECTIVES}

While our knowledge on plant MAP4Ks is gradually increasing, there is still very little known about them (Figure 1A). One of the main outstanding questions-also for non-plant MAP4Ks-is what their substrates are and if they thus can all be considered as true MAP4Ks. More and more evidence is accumulating that these MAP4Ks also have other targets than MAP3Ks (Plouffe et al., 2016; Xiong et al., 2016; Bae and Luo, 2018; Zhang et al., 2018; Jiang et al., 2019). And, there is-so far-no evidence that plant MAP4Ks act on MAP3Ks. Taken together, this might warrant revisiting the MAP $4 \mathrm{~K}$ name. Second, we lack a comprehensive overview of the MAP4Ks in the green lineage, which would facilitate defining common characteristics. Third, using current methodologies to explore kinase signaling (Zhang et al., 2016) is an essential next step to deepen our insight not only regarding the substrates, but also with respect to potential protein complexes that are formed. Fourth, the subcellular localization of MAP4Ks spans the plasma membrane, cytoplasm, and nucleus, but the precise role in these compartments has hardly been investigated.

\section{AUTHOR CONTRIBUTIONS}

LP and IS organized and wrote the manuscript.

\section{FUNDING}

LP was supported by a grant from the Chinese Scholarship Council.

\section{ACKNOWLEDGMENTS}

We thank Lam Dai Vu for critical comments on the manuscript.

Castells, E., Puigdomenech, P., and Casacuberta, J. M. (2006). Regulation of the kinase activity of the MIK GCK-like MAP4K by alternative splicing. Plant Mol. Biol. 61, 747-756. doi: 10.1007/s11103-006-0046-3

Champion, A., Picaud, A., and Henry, Y. (2004). Reassessing the MAP3K and MAP4K relationships. Trends Plant Sci. 9, 123-129. doi: 10.1016/ j.tplants.2004.01.005

Chen, Z., Raman, M., Chen, L., Lee, S. F., Gilman, A. G., and Cobb, M. H. (2003). TAO (thousand-and-one amino acid) protein kinases mediate signaling from carbachol to p38 mitogen-activated protein kinase and ternary complex factors. J. Biol. Chem. 278, 22278-22283. doi: 10.1074/jbc.M301173200 
Chen, R., Xie, R., Meng, Z., Ma, S., and Guan, K. L. (2019). STRIPAK integrates upstream signals to initiate the Hippo kinase cascade. Nat. Cell Biol. 21, 15651577. doi: 10.1038/s41556-019-0426-y

Cheng, Z., Li, J. F., Niu, Y., Zhang, X. C., Woody, O. Z., Xiong, Y., et al. (2015). Pathogen-secreted proteases activate a novel plant immune pathway. Nature 521, 213-216. doi: 10.1038/nature 14243

Chinchilla, D., Zipfel, C., Robatzek, S., Kemmerling, B., Nurnberger, T., Jones, J. D., et al. (2007). A flagellin-induced complex of the receptor FLS2 and BAK1 initiates plant defence. Nature 448, 497-500. doi: 10.1038/nature05999

Cho, S. K., Larue, C. T., Chevalier, D., Wang, H., Jinn, T.-L., Zhang, S., et al. (2008). Regulation of floral organ abscission in Arabidopsis thaliana. Proc. Natl. Acad. Sci. 105, 15629-15634.

Chuang, H. C., Wang, X., and Tan, T. H. (2016). MAP4K family kinases in immunity and inflammation. Adv. Immunol. 129, 277-314. doi: 10.1016/ bs.ai.2015.09.006

Colcombet, J., and Hirt, H. (2008). Arabidopsis MAPKs: a complex signalling network involved in multiple biological processes. Biochem. J. 413, 217-226. doi: 10.1042/BJ20080625

Couto, D., Niebergall, R., Liang, X., Bucherl, C. A., Sklenar, J., Macho, A. P., et al. (2016). The Arabidopsis protein phosphatase PP2C38 negatively regulates the central immune kinase BIK1. PLoS Pathog. 12, e1005811. doi: 10.1371/ journal.ppat.1005811

Dan, I., Watanabe, N. M., and Kusumi, A. (2001). The Ste20 group kinases as regulators of MAP kinase cascades. Trends Cell Biol. 11, 220-230. doi: 10.1016/ S0962-8924(01)01980-8

Dissmeyer, N., and Schnittger, A. (2011). The age of protein kinases. Plant Kinases. 779, 7-52. doi: 10.1007/978-1-61779-264-9_2

Galla, G., Zenoni, S., Marconi, G., Marino, G., Botton, A., Pinosa, F., et al. (2011). Sporophytic and gametophytic functions of the cell cycle-associated Mob1 gene in Arabidopsis thaliana L. Gene 484, 1-12. doi: 10.1016/j.gene.2011.05.009

Jiang, Y., Han, B., Zhang, H., Mariappan, K. G., Bigeard, J., Colcombet, J., et al. (2019). MAP4K4 associates with BIK1 to regulate plant innate immunity. EMBO Rep. 20, e47965. doi: 10.15252/embr.201947965

Jonak, C. (2002). Complexity, cross talk and integration of plant MAP kinase signalling. Curr. Opin. Plant Biol. 5, 415-424. doi: 10.1016/s1369-5266(02) 00285-6

Kinoshita, T., and Shimazaki, K. I. (1999). Blue light activates the plasma membrane $\mathrm{H}^{+}$-ATPase by phosphorylation of the C-terminus in stomatal guard cells. EMBO J. 18, 5548-5558. doi: 10.1093/emboj/18.20.5548

Kinoshita, T., Doi, M., Suetsugu, N., Kagawa, T., Wada, M., and Shimazaki, K.-i. (2001). Phot 1 and phot 2 mediate blue light regulation of stomatal opening. Nature 414, 65-660. doi: 10.1038/414656a

Kostaki, K. I., Coupel-Ledru, A., Bonnell, V. C., Gustavsson, M., Sun, P., McLaughlin, F. J., et al. (2020). Guard cells integrate light and temperature signals to control stomatal aperture. Plant Physiol. 182, 1404-1419. doi: $10.1104 /$ pp.19.01528

Krysan, P. J., and Colcombet, J. (2018). Cellular complexity in MAPK signaling in plants: Questions and emerging tools to answer them. Front. Plant Sci. 9:1674. doi: 10.3389/fpls.2018.01674

Lau, O. S., and Bergmann, D. C. (2012). Stomatal development: a plant's perspective on cell polarity, cell fate transitions and intercellular communication. Development 139, 3683-3692. doi: 10.1242/dev.080523

Leprince, A., Jouannic, S., Hamal, A., Kreis, M., and Henry, Y. (1999). Molecular characterisation of plant cDNAs BnMAP4K $\alpha 1$ and BnMAP4K $\alpha 2$ belonging to the GCK/SPS1 subfamily of MAP kinase kinase kinase kinas. Biochim. Biophys. Acta 1444 (1), 1-13. doi: 10.1016/s0167-4781(98)00246-2

Liu, Y., and Zhou, J. (2018). MAPping kinase regulation of ICE1 in freezing tolerance. Trends Plant Sci. 23, 91-93. doi: 10.1016/j.tplants.2017. 12.002

Llompart, B., Castells, E., Rio, A., Roca, R., Ferrando, A., Stiefel, V., et al. (2003). The direct activation of MIK, a germinal center kinase (GCK)-like kinase, by MARK, a maize atypical receptor kinase, suggests a new mechanism for signaling through kinase-dead receptors. J. Biol. Chem. 278, 48105-48411. doi: 10.1074/jbc.M307482200

Major, G., Daigle, C., Stafford-Richard, T., Tebbji, F., Lafleur, É., Caron, S., et al. (2009). Characterization of ScMAP4K1, a MAP kinase kinase kinase kinase involved in ovule, seed and fruit development in Solanum chacoense bitt. Plant Biol. 10, 27-46.
Meng, X., Shan, L., and He, P. (2015a). Stack heterotrimeric G proteins and MAPK cascades on a RACK. Mol. Plant 8, 1691-1693. doi: 10.1016/j.molp.2015.11.005

Meng, X., Chen, X., Mang, H., Liu, C., Yu, X., Gao, X., et al. (2015b). Differential function of Arabidopsis SERK family receptor-like kinases in stomatal patterning. Curr. Biol. 25, 2361-2372. doi: 10.1016/j.cub.2015.07.068

Miller, C. J., Lou, H. J., Simpson, C., van de Kooij, B., Ha, B. H., Fisher, O. S., et al. (2019). Comprehensive profiling of the Ste20 kinase family defines features essential for selective substrate targeting and signaling output. PLoS Biol. 17, e2006540. doi: 10.1371/journal.pbio.2006540

Mishra, N. S., Tuteja, R., and Tuteja, N. (2006). Signaling through MAP kinase networks in plants. Arch. Biochem. Biophys. 452, 55-68. doi: 10.1016/ j.abb.2006.05.001

Pan, D. (2010). The Hippo signaling pathway in development and cancer. Dev. Cell. 19, 491-505. doi: 10.1016/j.devcel.2010.09.011

Pinosa, F., Begheldo, M., Pasternak, T., Zermiani, M., Paponov, I. A., Dovzhenko, A., et al. (2013). The Arabidopsis thaliana Mob1A gene is required for organ growth and correct tissue patterning of the root tip. Ann. Botany 112, 18031814. doi: $10.1093 / \mathrm{aob} / \mathrm{mct} 235$

Plouffe, S. W., Meng, Z., Lin, K. C., Lin, B., Hong, A. W., Chun, J. V., et al. (2016). Characterization of Hippo pathway components by gene inactivation. Mol. Cell. 64, 993-1008. doi: 10.1016/j.molcel.2016.10.034

Schnabel, J., Hombach, P., Waksman, T., Giuriani, G., Petersen, J., and Christie, J. M. (2018). A chemical genetic approach to engineer phototropin kinases for substrate labeling. J. Biol. Chem. 293, 5613-5623. 10.1074/ jbc.RA118.001834.

Sopena-Torres, S., Jorda, L., Sanchez-Rodriguez, C., Miedes, E., Escudero, V., Swami, S., et al. (2018). YODA MAP3K kinase regulates plant immune responses conferring broad-spectrum disease resistance. New Phytol. 218, 661-680. doi: 10.1111/nph.15007

Stone, J. M., and Walker, J. C. (1995). Plant protein kinase families and signal transduction. Plant Cell Physiol. 108, 451-457. doi: 10.1104/pp.108.2.451

$\mathrm{Su}, \mathrm{J}$., Xu, J., and Zhang, S. (2015). Rack1, scaffolding a heterotrimeric G protein and a MAPK cascade. Trends Plant Sci. 20, 405-407. doi: 10.1016/ j.tplants.2015.05.002

Takemiya, A., and Shimazaki, K. (2016). Arabidopsis phot1 and phot2 phosphorylate BLUS1 kinase with different efficiencies in stomatal opening. J. Plant Res. 129, 167-174. doi: 10.1007/s10265-015-0780-1

Takemiya, A., Kinoshita, T., Asanuma, M., and Shimazaki, K.-i. (2006). Protein phosphatase 1 positively regulates stomatal opening in response to blue light in Vicia faba. Proc. Natl. Acad. Sci. 103, 13549-13554. doi: 10.1073/pnas. 0602503103

Takemiya, A., Sugiyama, N., Fujimoto, H., Tsutsumi, T., Yamauchi, S., Hiyama, A., et al. (2013a). Phosphorylation of BLUS1 kinase by phototropins is a primary step in stomatal opening. Nat. Commun. 4, 1-8. doi: 10.1038/ ncomms3094

Takemiya, A., Yamauchi, S., Yano, T., Ariyoshi, C., and Shimazaki, K. (2013b). Identification of a regulatory subunit of protein phosphatase 1 which mediates blue light signaling for stomatal opening. Plant Cell Physiol. 54, 24-35. doi: $10.1093 / \mathrm{pcp} / \mathrm{pcs} 073$

Wang, D., Harper, J. F., and Gribskov, M. (2003). Systematic trans-genomic comparison of protein kinases between Arabidopsis and Saccharomyces cerevisiae. Plant Physiol. 132, 2152-2165. doi: 10.1104/pp.103.021485

Wang, H., Chevalier, D., Larue, C., Ki Cho, S., and Walker, J. C. (2007). The protein phosphatases and protein kinases of Arabidopsis thaliana. Arabidopsis Book 5, e0106. doi: 10.1199/tab.0106

Wu, C., Whiteway, M., Thomas, D., and Leberer, E. (1995). Molecular characterization of Ste20p, a potential mitogen-activated protein or extracellular signal-regulated kinase kinase (MEK) kinase kinase from Saccharomyces cerevisiae. J. Biol. Chem. 270, 15984-15992. doi: 10.1074/ jbc.270.27.15984

Xiong, J., Cui, X., Yuan, X., Yu, X., Sun, J., and Gong, Q. (2016). The Hippo/Ste20 homolog SIK1 interacts with MOB1 to regulate cell proliferation and cell expansion in Arabidopsis. J. Exp. Botany 67, 1461-1675. doi: 10.1093/jxb/erv538

$\mathrm{Xu}$, J., and Zhang, S. (2015). Mitogen-activated protein kinase cascades in signaling plant growth and development. Trends Plant Sci. 20, 56-64. doi: 10.1016/j.tplants.2014.10.001

Yan, H., Zhao, Y., Shi, H., Li, J., Wang, Y., and Tang, D. (2018). BRASSINOSTEROID-SIGNALING KINASE1 phosphorylates MAPKKK5 to 
regulate immunity in Arabidopsis. Plant Physiol. 176, 2991-3002. doi: 10.1104/ pp.17.01757

Yu, M. H., Zhao, Z. Z., and He, J. X. (2018). Brassinosteroid signaling in plant-microbe interactions. Int. J. Mol. Sci. 19, 4091. doi: 10.3390/ijms 19124091

Zhang, T., Chen, S., and Harmon, A. C. (2016). Protein-protein interactions in plant mitogen-activated protein kinase cascades. J. Exp. Botany 67 (3), 607-618. doi: 10.1093/jxb/erv508

Zhang, M., Chiang, Y. H., Toruno, T. Y., Lee, D., Ma, M., Liang, X., et al. (2018). The MAP4 kinase SIK1 ensures robust extracellular ROS burst and antibacterial immunity in plants. Cell Host Microbe 24, 379-391. doi: 10. 1016/j.chom.2018.08.007

Zhao, C., Wang, P., Si, T., Hsu, C. C., Wang, L., Zayed, O., et al. (2017). MAP kinase cascades regulate the cold response by modulating ICE1 protein stability. Dev. Cell. 43, 618-629 e615. doi: 10.1016/j.devcel.2017.09.024
Zhu, Q., Shao, Y., Ge, S., Zhang, M., Zhang, T., Hu, X., et al. (2019). A MAPK cascade downstream of IDA-HAE/HSL2 ligand-receptor pair innbsp;lateral root emergence. Nat. Plants 5, 414-423. doi: 10.1038/ s41477-019-0396-x

Conflict of Interest: The authors declare that the research was conducted in the absence of any commercial or financial relationships that could be construed as a potential conflict of interest.

Copyright (C) 2020 Pan and De Smet. This is an open-access article distributed under the terms of the Creative Commons Attribution License (CC BY). The use, distribution or reproduction in other forums is permitted, provided the original author(s) and the copyright owner(s) are credited and that the original publication in this journal is cited, in accordance with accepted academic practice. No use, distribution or reproduction is permitted which does not comply with these terms. 